\title{
Dynamic Characteristics of Deeply Buried Spherical Biogas Digesters in Viscoelastic Soils
}

\author{
Hongwei Hou, ${ }^{1,2}$ Shihu Gao $\mathbb{D}^{3},{ }^{3}$ Qianqian Guo, ${ }^{4}$ Long Chen, ${ }^{4}$ Bing Wu $\mathbb{D},{ }^{4}$ and Lei Wang ${ }^{5}$ \\ ${ }^{1}$ Zhejiang Academy of Building Research and Design Co. Ltd., Hangzhou, Zhejiang 310000, China \\ ${ }^{2}$ College of Civil Engineering and Architecture, Zhejiang University, Hangzhou, Zhejiang 310000, China \\ ${ }^{3}$ College of Civil Engineering and Architecture, Wenzhou University, Wenzhou, Zhejiang 325035, China \\ ${ }^{4}$ Key Laboratory of Ministry of Education for Geomechanics and Embankment Engineering, Hohai University, Nanjing 210098, China \\ ${ }^{5}$ Shanghai City Construction and Design Research Institute, No. 1170 Tibet South Road, Huangpu District, Shanghai, China
}

Correspondence should be addressed to Shihu Gao; lzccgsh@163.com

Received 10 January 2018; Revised 2 May 2018; Accepted 10 May 2018; Published 18 October 2018

Academic Editor: Zhi-Yuan Sun

Copyright (c) 2018 Hongwei Hou et al. This is an open access article distributed under the Creative Commons Attribution License, which permits unrestricted use, distribution, and reproduction in any medium, provided the original work is properly cited.

\begin{abstract}
The harmonic vibration characteristics of a deeply buried spherical methane tank in viscoelastic soil subjected to cyclic loading in the frequency domain are investigated. The dynamic behavior of the soil is described based on the theory of fractional derivatives. By introducing potential functions, the closed-form expressions for the displacement and the stress of the viscoelastic soil surrounding the deeply buried spherical methane tank are obtained. Two die structures are considered: a homogeneous elastic medium and a shell structure. Based on the theory of elastic motion and the Flügge theory, analytic solutions for the dynamic responses of the spherical methane tank in a fractional-derivative viscoelastic soil are derived explicitly. Analytic solution expressions of the undetermined coefficients are determined by using the continuum boundary conditions. The system dynamic responses to the homogeneous elastic medium and the shell structure and the influences of the parameters of the fractional derivative, soil, and die on the dynamic characteristic of the system are compared and analyzed. The results indicate a significant difference between the dynamic responses of the die structures for the two models.
\end{abstract}

\section{Introduction}

Not only has an underground, deeply buried spherical digester the advantages of a small spherical mouth, good airtightness, and high gas production but also the fermentation of biogas slurry does not produce a crust and the structure is not affected by the external ambient temperature [1]. Therefore, spherical underground digesters are widely used and are promoted in China's rural areas.

The vibration response of biogas digesters has wide applications in the fields of seismic resistance, explosion-resistant design, and structural stability of the die. Forrestal and Sagartz studied the dynamic responses of thin-walled spherical shells and cylindrical shells in homogeneous elastic soil under the action of heavy loads [2]. Duffey obtained the transient dynamic response of viscoelastic and viscous-plastic spherical die structures under impulsive loading by using a Laplace transform technique [3]. Glenn regarded the soil as a linear elastic medium and deduced the vibration response of a deeply buried spherical digester under heavy loads and pulse loads [4]. The transient response of a spherical cavity has been studied under explosive loading by using displacement as an unknown quantity [5].

Many experimental studies have been carried out to investigate the clay behavior under dynamic loading; Lin Guo studied the deformation behavior of saturated soft soil under long-term cyclic loading and established two equations for the prediction of long-term resilient modulus and permanent strain $[6,7]$. Strain and pore pressure development on soft marine soil have been studied under dynamic loadings and an empirical formula is established to predict the long-term permanent strain [8]. Chuan Gu studied the influence of cyclic loading history on small strain shear modulus of saturated soils by triaxial test; test results show that both cyclic strain history and cyclic stress history will induce reduction of Gmax compared to the corresponding Gmax values with 
noncyclic loading effects at the same effective stress [9]. Dynamic modulus characteristics of saturated soils under variable confining pressure have been studied by cyclic triaxial tests; tests results shows that both the phase differences and ratios between the cyclic deviatoric stress and cyclic confining pressure exerted a constant and pronounced influence on dynamic modulus [10-12].

Studies of the viscoelastic properties of soils have shown that a fractional-derivative constitutive model is well suited for the characterization of the soil's creep properties [13]. However, based on the fractional-derivative model, the dynamic equation is a singular integral-partial differential equation and its theoretical analysis and numerical calculation present many difficulties [14] and there have been few reports on the use of this approach for the study of soil dynamic characteristics [15]. What is more, the above studies ignore the thickness of the mold structure. In this paper, the soil element is regarded as a viscoelastic element with fractional-derivative properties and the exact expression of the displacement and stress of the infinite fractional-derivative viscoelastic soil surrounding the biogas digester is deduced based on the viscoelastic theory. In our research, the thickness of the mold structure also can be considered.

The gas fermentation and user consumption cause repeated changes in pressure in the pool and this pressure is uniformly applied to the inner side of the pool wall and is equivalent to the periodic load [16]. In this study, the soil body is regarded as a viscoelastic body with fractional-derivative properties and the exact expression of the displacement and stress of the infinite fractional-derivative viscoelastic soil surrounding the biogas digester is deduced based on the viscoelastic theory. Secondly, the die structure is considered a homogeneous elastic medium and thin-walled shell structure and the analytics solution for the vibration response of the die structure is obtained based on the theory of elastic motion [17] and the Flügge theory [18]. Finally, the concrete expression of the relevant undetermined coefficients is obtained according to the boundary conditions of displacement and stress in the mold structure and the contact surface of the soil body. A comparison of the results of this study and existing data demonstrates the correctness of the assumptions of this study. Finally, the influences of the parameters of the fractional derivative on the vibration response of the system are investigated, providing a theoretical basis for the design and construction of underground spherical digesters.

\section{Mathematical Model}

Because the spherical digester is deeply embedded in the soil, it is assumed that there is a spherical digester with an inner radius $R_{1}$ and an outer radius $R_{2}$, as shown in Figure 1; the distance from the center to the middle surface is $a$. The spherical digester has a circular frequency $\omega$ of a uniform radial harmony excitation load $\left(q_{0} \mathrm{e}^{\mathrm{i} \omega \mathrm{t}}\right)$, where $i$ is the imaginary unit. The shear modulus parameters of the soil and die structure are $G^{S}$ and $G^{\mathrm{L}}$, respectively, the material density parameters are $\rho^{\mathrm{S}}$ and $\rho^{\mathrm{L}}$, respectively, and the Poisson's ratios of the soil and the model are $v^{S}$ and $v^{\mathrm{L}}$, respectively. The mold

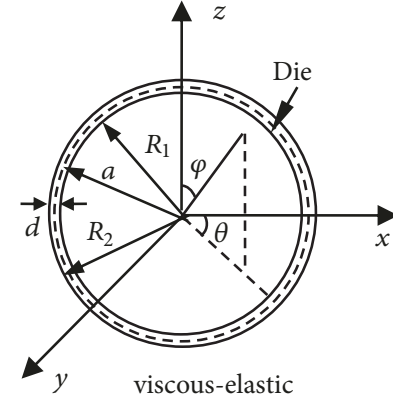

FIgURE 1: A spherical biogas digester in viscoelastic soil.

and the soil interface are in complete and close contact to meet the stress and displacement continuity.

\section{Establish and Solution of the Soil Control Equation}

In the case of spherical symmetry, the stress-displacement constitutive relationship of the constitutive model with the fractional derivative is [11]

$$
\begin{aligned}
(1 & \left.+\tau_{\varepsilon}^{\alpha} D^{\alpha}\right) \sigma_{r}^{\mathrm{S}} \\
& =\left(1+\tau_{\sigma}^{\alpha} D^{\alpha}\right)\left[\lambda^{\mathrm{S}}\left(\frac{\partial u_{r}^{\mathrm{S}}}{\partial r}+\frac{2 u_{r}^{\mathrm{S}}}{r}\right)+2 \mu^{\mathrm{S}} \frac{\partial u_{r}^{\mathrm{S}}}{\partial r}\right] \\
(1 & \left.+\tau_{\varepsilon}^{\alpha} D^{\alpha}\right) \sigma_{\theta}^{\mathrm{S}} \\
& =\left(1+\tau_{\sigma}^{\alpha} D^{\alpha}\right)\left[\lambda^{\mathrm{S}}\left(\frac{\partial u_{r}^{\mathrm{S}}}{\partial r}+\frac{2 u_{r}^{\mathrm{S}}}{r}\right)+2 \mu^{\mathrm{S}} \frac{u_{r}^{\mathrm{S}}}{r}\right] \\
\left(1+\tau_{\varepsilon}^{\alpha} D^{\alpha}\right) \sigma_{\varphi}^{\mathrm{S}} & \left(1+\tau_{\sigma}^{\alpha} D^{\alpha}\right)\left[\lambda^{\mathrm{S}}\left(\frac{\partial u_{r}^{\mathrm{S}}}{\partial r}+\frac{2 u_{r}^{\mathrm{S}}}{r}\right)+2 \mu^{\mathrm{S}} \frac{u_{r}^{\mathrm{S}}}{r}\right]
\end{aligned}
$$

Where $\sigma_{\mathrm{r}}$ is radial stress of soil layer, $\sigma_{\theta}$ is circumferential stress of soil layer, $\sigma_{\varphi}$ is shear stress of soil layer, $\sigma$ is stress tensor of soil, $\varepsilon$ is strain tensor of soil, $\tau_{\varepsilon}$ and $\tau_{\sigma}$ are constitutive model parameters, $\lambda^{\mathrm{s}}$ and $\mu^{\mathrm{s}}$ are Lame constant of soil skeleton and $\lambda^{\mathrm{S}}=2 \nu^{\mathrm{S}} /\left(1-2 \nu^{\mathrm{S}}\right)$, and $\nu$ is Poisson's ratio. $\mathrm{D}^{\alpha}=\mathrm{d}^{\alpha} / \mathrm{dt}^{\alpha}$ is $\alpha$-step Riemann-Liouville fractional derivative, which is defined as

$$
D^{\alpha}[x(t)]=\frac{1}{\Gamma(1-\alpha)} \frac{d}{d t} \int_{0}^{t} \frac{x(\tau)}{(t-\tau)^{\alpha}} d \tau
$$

in which $\Gamma(u)=\int_{0}^{\infty} t^{u-1} e^{-u} d t$ is Gamma Function.

Under the action of dynamic load, according to the theory of elasticity, the dynamic equation [16] of soil is as follows.

$$
\frac{\partial \sigma_{r}^{\mathrm{S}}}{\partial r}+\frac{2 \sigma_{r}^{\mathrm{S}}-\sigma_{\theta}^{\mathrm{S}}-\sigma_{\varphi}^{\mathrm{S}}}{r}=\rho^{\mathrm{S}} \frac{\partial^{2} u_{r}^{\mathrm{S}}}{\partial t^{2}}
$$

Substituting (1) into (3), we obtain

$$
\left(\lambda^{\mathrm{S}}+2 \mu^{\mathrm{S}}\right) \frac{1+\tau_{\sigma}^{\alpha} D^{\alpha}}{1+\tau_{\varepsilon}^{\alpha} D^{\alpha}} \frac{\partial}{\partial r}\left(\frac{\partial u_{r}^{\mathrm{S}}}{\partial r}+\frac{2 u_{r}^{\mathrm{S}}}{r}\right)=\rho^{\mathrm{S}} \frac{\partial^{2} u_{r}^{\mathrm{S}}}{\partial t^{2}}
$$


Introduce potential function

$$
u_{r}^{\mathrm{S}}=\frac{\partial}{\partial r}\left(\frac{\varphi^{\mathrm{S}}(r, t)}{r}\right)
$$

For steady-state vibration, let

$$
\begin{aligned}
& u_{r}^{\mathrm{S}}=R_{2} U_{\eta}^{\mathrm{S}} e^{\mathrm{i} \omega t}, \\
& \varphi^{\mathrm{S}}=R_{2}^{3} \bar{\varphi}^{\mathrm{S}} e^{\mathrm{i} \omega t}
\end{aligned}
$$
lows:

Introduce dimensionless quantity and constant as fol-

$$
\begin{aligned}
\eta & =\frac{r}{R_{2}}, \\
\lambda & =\frac{\omega R_{2}}{V^{S}}, \\
T_{\sigma} & =\frac{\tau_{\sigma} V^{S}}{R_{2}}, \\
T_{\varepsilon} & =\frac{\tau_{\varepsilon} V^{S}}{R_{2}}, \\
\eta_{0} & =1-\delta, \\
\delta & =\frac{d}{R_{2}} .
\end{aligned}
$$

Substituting (5), (6), and (7) into (4), we obtain (8) as follows:

$$
\frac{d^{2} \bar{\varphi}^{\mathrm{S}}}{d \eta^{2}}-\beta^{2} \bar{\varphi}^{\mathrm{S}}=0
$$

where

$$
\beta^{2}=-\frac{1+T_{\varepsilon}^{\alpha}(\mathrm{i} \lambda)^{\alpha}}{1+T_{\sigma}^{\alpha}(\mathrm{i} \lambda)^{\alpha}} \frac{\left(1-2 v^{\mathrm{S}}\right) \lambda^{2}}{2\left(1-v^{\mathrm{S}}\right)}
$$

According to (8), we obtain

$$
\bar{\varphi} S=A e^{-\beta \eta}+B e^{\beta \eta}
$$

where A and B are undetermined coefficient; they are determined by boundary conditions. According to boundary conditions $r \longrightarrow \infty, u_{r}^{\mathrm{S}}=0$, we obtain $\mathrm{B}=0$,

$$
\bar{\varphi}^{S}=A e^{-\beta \eta}
$$

Substituting (5) into (11), we obtain (12) as follows:

$$
U_{\eta}^{S}=-\left(\frac{1}{\eta^{2}}+\frac{\beta}{\eta}\right) e^{-\beta \eta} A
$$

Substituting (12) into (1), we obtain (13) as follows:

$$
\begin{aligned}
\sigma_{r}^{\mathrm{S}} & =\mu^{\mathrm{S}} \frac{1+T_{\sigma}^{\alpha}(\mathrm{i} \lambda)}{1+T_{\varepsilon}^{\alpha}(\mathrm{i} \lambda)} \\
& \cdot e^{\mathrm{i} \omega t}\left[\frac{2 v^{\mathrm{S}}}{1-2 v^{\mathrm{S}}} \frac{\beta^{2}}{\eta}+2\left(\frac{2}{\eta^{3}}+\frac{2 \beta}{\eta^{2}}+\frac{\beta^{2}}{\eta}\right)\right] e^{-\beta \eta} A \\
\sigma_{\theta}^{\mathrm{S}} & =\mu^{\mathrm{S}} \frac{1+T_{\sigma}^{\alpha}(\mathrm{i} \lambda)}{1+T_{\varepsilon}^{\alpha}(\mathrm{i} \lambda)} e^{\mathrm{i} \omega t}\left[\frac{2 v^{\mathrm{S}}}{1-2 v^{\mathrm{S}}} \frac{\beta^{2}}{\eta}-2\left(\frac{1}{\eta^{3}}+\frac{\beta}{\eta^{2}}\right)\right] \\
& \cdot e^{-\beta \eta} A
\end{aligned}
$$

\section{Establishment and Solution of Die Structure Motion Equation}

4.1. Die Structure Equation of Type 1. We considered the mold structure of the spherical digester as a homogeneous elastic medium and assumed that the mold structure and soil interface are in complete and close contact with no relative slip to satisfy the stress and displacement continuity. Under the action of a dynamic load, the motion equation of the die junction is as follows [16].

$$
\frac{\partial \sigma_{r}^{\mathrm{L}}}{\partial r}+\frac{2 \sigma_{r}^{\mathrm{L}}-\sigma_{\theta}^{\mathrm{L}}-\sigma_{\varphi}^{\mathrm{L}}}{r}=\rho^{\mathrm{L}} \frac{\partial^{2} u_{r}^{\mathrm{L}}}{\partial t^{2}}
$$

Constitutive model is

$$
\begin{aligned}
& \sigma_{r}^{\mathrm{L}}=\lambda^{\mathrm{L}}\left(\frac{\partial u_{r}^{\mathrm{L}}}{\partial r}+\frac{2 u_{r}^{\mathrm{L}}}{r}\right)+2 \mu^{\mathrm{L}} \frac{\partial u_{r}^{\mathrm{L}}}{\partial r} \\
& \sigma_{\theta}^{\mathrm{L}}=\lambda^{\mathrm{L}}\left(\frac{\partial u_{r}^{\mathrm{L}}}{\partial r}+\frac{2 u_{r}^{\mathrm{L}}}{r}\right)+2 \mu^{\mathrm{L}} \frac{u_{r}^{\mathrm{L}}}{r} \\
& \sigma_{\varphi}^{\mathrm{L}}=\lambda^{\mathrm{L}}\left(\frac{\partial u_{r}^{\mathrm{L}}}{\partial r}+\frac{2 u_{r}^{\mathrm{L}}}{r}\right)+2 \mu^{\mathrm{L}} \frac{u_{r}^{\mathrm{L}}}{r}
\end{aligned}
$$

Substituting (15) into (14), we obtain (16) as follows:

$$
\left(\lambda^{\mathrm{L}}+2 \mu^{\mathrm{L}}\right) \frac{\partial}{\partial r}\left(\frac{\partial u_{r}^{\mathrm{L}}}{\partial r}+\frac{2 u_{r}^{\mathrm{L}}}{r}\right)=\rho^{\mathrm{L}} \frac{\partial^{2} u_{r}^{\mathrm{L}}}{\partial t^{2}}
$$

For steady-state vibration, let $u_{r}^{\mathrm{L}}=R_{2} U_{\eta}^{\mathrm{L}} e^{\mathrm{i} \omega t}, \varphi^{\mathrm{L}}=$ $R_{2}^{3} \bar{\varphi}^{\mathrm{L}} e^{\mathrm{i} \omega t}$. Introduce displacement potential function

$$
u_{r}^{\mathrm{L}}=\frac{\partial}{\partial r}\left(\frac{\varphi^{\mathrm{L}}(r, t)}{r}\right)
$$

Substituting (17) into (16), we obtain

$$
\frac{d^{2} \bar{\varphi}^{\mathrm{L}}}{d \eta^{2}}-q^{2} \bar{\varphi}^{\mathrm{L}}=0
$$


where

$$
\begin{aligned}
G^{\mathrm{SL}} & =\frac{G^{\mathrm{S}}}{G^{\mathrm{L}}}, \\
\rho^{\mathrm{LS}} & =\frac{\rho^{\mathrm{L}}}{\rho^{\mathrm{S}}}, \\
q^{2} & =-\frac{\left(1-2 v^{\mathrm{L}}\right) \lambda^{2} \rho^{\mathrm{LS}} G^{\mathrm{SL}}}{2\left(1-v^{\mathrm{L}}\right)}
\end{aligned}
$$

According to (18), we obtain

$$
\bar{\varphi}^{\mathrm{L}}=C_{5} e^{-q \eta}+C_{6} e^{q \eta}
$$

where $\mathrm{C}_{5}$ and $\mathrm{C}_{6}$ are undetermined coefficient; they are determined by boundary conditions. Substituting (20) into (17), we obtain

$$
U_{\eta}^{\mathrm{L}}=-\left(\frac{1}{\eta^{2}}+\frac{q}{\eta}\right) e^{-q \eta} C_{5}-\left(\frac{1}{\eta^{2}}-\frac{q}{\eta}\right) e^{q \eta} C_{6}
$$

According to (15), we obtain radial stress amplitude

$$
\begin{aligned}
\sigma_{r}^{\mathrm{L}}= & \left(\frac{2 v^{\mathrm{L}} G^{\mathrm{L}}}{1-2 v^{\mathrm{L}}} \frac{q^{2}}{\eta}+2 G^{\mathrm{L}} \vartheta_{1}\right) e^{-q \eta} e^{\mathrm{i} \omega t} C_{5} \\
& +\left(\frac{2 v^{\mathrm{L}} G^{\mathrm{L}}}{1-2 v^{\mathrm{L}}} \frac{q^{2}}{\eta}+2 G^{\mathrm{L}} \vartheta_{2}\right) e^{q \eta} e^{\mathrm{i} \omega t} C_{6}
\end{aligned}
$$

where

$$
\begin{aligned}
& \vartheta_{1}=\frac{2}{\eta^{3}}+\frac{2 q}{\eta^{2}}+\frac{q^{2}}{\eta}, \\
& \vartheta_{2}=\frac{2}{\eta^{3}}-\frac{2 q}{\eta^{2}}+\frac{q^{2}}{\eta}
\end{aligned}
$$

Satisfy the boundary conditions as follows:

(1) Displacement continuous in the mold structure and soil interface $\left(r=R_{2}, \eta=1\right)$; that is,

$$
u_{r}^{\mathrm{S}}=u_{r}^{\mathrm{L}} \quad r=R_{2}
$$

(2) Stress continuity in the mold structure and soil interface $\left(r=R_{2}, \eta=1\right)$; that is,

$$
\sigma_{r}^{\mathrm{S}}=\sigma_{r}^{\mathrm{L}} \quad r=R_{2}
$$

(3) Internal hemorrhage loading equal to the radial stress in the mold structure and soil interface $\left(r=R_{1}, \eta=1\right)$ of the die structure; that is,

$$
\sigma_{r}^{\mathrm{L}}=q_{0} e^{\mathrm{i} \omega t} \quad r=R_{1}
$$

According to (24) (26), we obtain the actual formulas of the undetermined coefficients $A, C_{5}, C_{6}$,

$$
\begin{aligned}
C_{6} & =\frac{a_{2} b_{1}-a_{1} b_{2}}{e_{1}\left(a_{1} b_{3}-a_{3} b_{1}\right)+e_{2}\left(a_{2} b_{1}-a_{1} b_{2}\right)} Q \\
C_{5} & =\frac{Q}{e_{1}}-\frac{e_{2}}{e_{1}} C_{6} \\
A & =-\frac{a_{2}}{a_{1}} C_{5}-\frac{a_{3}}{a_{1}} C_{6},
\end{aligned}
$$

where

$$
\begin{aligned}
a_{1} & =-(1+\beta) e^{-\beta}, \\
a_{2} & =(1+q) e^{-q}, \\
a_{3} & =(1-q) e^{q}, \\
b_{1} & =G^{\mathrm{SL}} \\
& \cdot \frac{1+T_{\sigma}^{\alpha}(\mathrm{i} \lambda)^{\alpha}}{1+T_{\varepsilon}^{\alpha}(\mathrm{i} \lambda)^{\alpha}}\left[\frac{2 v^{\mathrm{S}} \beta^{2} e^{-\beta}}{1-2 v^{\mathrm{S}}}+2 e^{-\beta}\left(2+2 \beta+\beta^{2}\right)\right], \\
b_{2}= & -\left[\frac{2 v^{\mathrm{L}} q^{2}}{1-2 v^{\mathrm{L}}}+2\left(2+2 q+q^{2}\right)\right] e^{-q}, \\
b_{3}= & -\left[\frac{2 v^{\mathrm{L}} q^{2}}{1-2 v^{\mathrm{L}}}+2\left(2-2 q+q^{2}\right)\right] e^{q}, \\
e_{1}= & {\left[\frac{2 v^{\mathrm{L}} q^{2}}{\left(1-2 v^{\mathrm{L}}\right) \eta_{0}}+2\left(\frac{2}{\eta_{0}^{3}}+\frac{2 q}{\eta_{0}^{2}}+\frac{q^{2}}{\eta_{0}}\right)\right] e^{-q \eta_{0}}, } \\
e_{2}= & {\left[\frac{2 v^{\mathrm{L}} q^{2}}{\left(1-2 v^{\mathrm{L}}\right) \eta_{0}}+2\left(\frac{2}{\eta_{0}^{3}}-\frac{2 q}{\eta_{0}^{2}}+\frac{q^{2}}{\eta_{0}}\right)\right] e^{q \eta_{0}} . }
\end{aligned}
$$

4.2. Die Structure Equation of Type 2. Since the thickness of the die structure is much smaller than the radius of the spherical digester, it can be assumed that the mold structure has little effect on the interface $r=R_{2}$ of the soil and the middle surface radius [6] $r=a$. According to the Hamilton principle, the motion equation of the die structure [7] is as follows:

$$
u_{r}^{\mathrm{L}} \frac{2 G^{\mathrm{L}} d}{\left(1-v^{\mathrm{L}}\right) R_{2}^{2}}+\rho^{\mathrm{L}} d \frac{\partial^{2} u_{r}^{\mathrm{L}}}{\partial t^{2}}=Q_{a}
$$

where $Q_{a}$ is net pressure.

For steady-state vibration, we assumed

$$
\begin{aligned}
u_{r}^{\mathrm{L}} & =R_{2} U_{\eta}^{\mathrm{L}} e^{\mathrm{i} \omega t}, \\
Q_{a} & =G^{\mathrm{S}} \overline{\mathrm{Q}}_{a} e^{\mathrm{i} \omega t} \\
G^{\mathrm{SL}} & =\frac{G^{\mathrm{S}}}{G^{\mathrm{L}}}, \\
\rho^{\mathrm{LS}} & =\frac{\rho^{\mathrm{L}}}{\rho^{\mathrm{S}}}
\end{aligned}
$$

Meanwhile, satisfy the boundary conditions as follows:

(1) Stress compatibility in the mold structure and soil interface $\left(r=R_{2}, \eta=1\right)$; that is,

$$
G^{\mathrm{SL}} \overline{\mathrm{Q}}_{a} e^{\mathrm{i} \omega t}=Q e^{\mathrm{i} \omega t}-\frac{\sigma_{r}^{\mathrm{S}}}{G^{\mathrm{L}}}
$$

(2) Displacement continuous in the mold structure and soil interface $\left(r=R_{2}, \eta=1\right)$; that is,

$$
u_{r}^{\mathrm{S}}=u_{r}^{\mathrm{L}} \quad r=R_{2}
$$




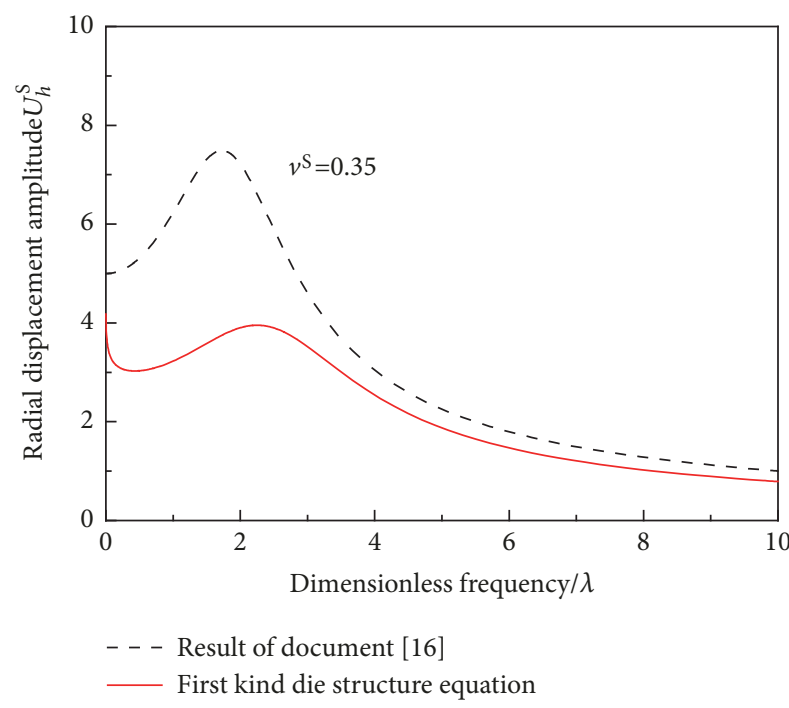

(a) Radial displacement amplitude

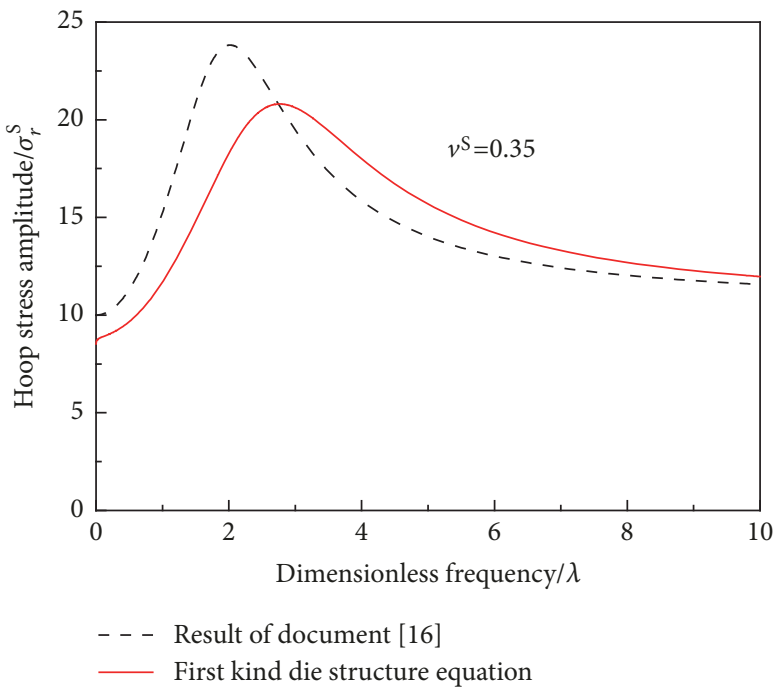

(b) Hoop stress amplitude

FIGURE 2: Comparison between present results and [16] results.

Substituting (29) and (13) into (31) and (32), we obtain

A

$$
=\frac{Q}{G^{\mathrm{SL}}\left(\left(1+T_{\sigma}^{\alpha}(\mathrm{i} \lambda)^{\alpha}\right) /\left(1+T_{\varepsilon}^{\alpha}(\mathrm{i} \lambda)^{\alpha}\right)\right) \vartheta_{3}-\vartheta_{4}(1+\beta) e^{-\beta}}
$$

where

$$
\begin{aligned}
& \vartheta_{3}=\frac{2 v^{\mathrm{S}} \beta^{2}}{1-2 v^{\mathrm{S}}} e^{-\beta}+2 e^{-\beta}\left(2+2 \beta+\beta^{2}\right) \\
& \vartheta_{4}=\frac{2 \delta}{1-2 v^{\mathrm{L}}}-\rho^{\mathrm{LS}} G^{\mathrm{SL}} \delta \lambda^{2}
\end{aligned}
$$

\section{Comparison with Existing Results}

In order to verify the correctness of the calculation, we compared the results with existing results, which include the ideal elastic model of [16], the classical elastic constitutive model [17], and classical viscoelastic constitutive model [18].

The ideal elastic model of [16] ignores the influence of the die structure firstly. Besides, it establishes the dynamic characteristics of the spherical digester in a general and ideal elastic soil. Substituting the equation for the classical elastic constitutive method into (3) and assuming that the material parameters of the soil are the same as in the die structure, we determined that the results of this study were in agreement with other published solutions [16]. The parameter values were as follows: $\eta=1, \mathrm{vS}=0.35, \rho \mathrm{LS}=1.5, \mathrm{GSL}=0.05, \mathrm{vL}=0.25$, $\delta=0.005, \mathrm{~T} \sigma / \mathrm{T} \mathcal{E}=3, \mathrm{~T} \mathcal{E}=10$, and $\alpha=0.5$. Figure 2 shows a curve for the radial displacement and hoop stress amplitude with a dimensionless frequency for the two models of a uniform elastic medium. It can be seen that, in [16], the resonance effect is significantly greater for the model than for the homogeneous elastic dielectric model and that the base frequency is also higher than the base frequency of the resonance effect of the system. There are two main reasons for this: firstly, the author uses the fractional-derivative constitutive model to describe the soil viscosity and [16] uses a general and ideal elastic soil body; secondly, the author considers the influence of the die structure which has a greater stiffness than the soil body.

Thirdly, the fractional-derivative constitutive model used in this study is compared with the classical elastic model [17] and the classical viscoelastic constitutive model [18]. Substituting the classical elastic constitutive model and classical viscoelastic constitutive model used in the literature [19] into the soil equation of motion (3), respectively, changes the results of $[19,20]$. Figure 3 shows the changes in the curves of the radial displacement amplitude and circumference displacement amplitude as a result of the changing frequency of the three models. It can be seen that, for the elastic model in [20], the peak value of the displacement amplitude of the soil is maximum; the resonance effect is clear and the resonance phenomenon decreases with increasing. The peak value of the classical viscoelastic model used in [19] is the minimum, while, for the fractional-derivative viscoelastic model, it is in the middle.

\section{Analysis of Example and Graphic Analysis}

The effects of the shear modulus ratio $G^{\mathrm{SL}}$ of the soil and die, the Poisson ratio $v^{\mathrm{s}}$ of the soil material, the fractionalderivative order $\alpha$, the material parameter ratio $T_{\sigma} / T_{\varepsilon}$ on the radial displacement, and the hoop stress amplitude were investigated.

Figure 4 shows that the values of the radial displacement and circumferential stress amplitude change with the frequency $\mathrm{f}$ as the shear modulus ratio $G^{\mathrm{SL}}$ of the soil body and die structure variation change. It is evident that when the modulus ratio $G^{\text {SL }}$ is comparatively small, the displacement amplitudes are very different for the model and the second type of mold model and the resonance effect produced by the 


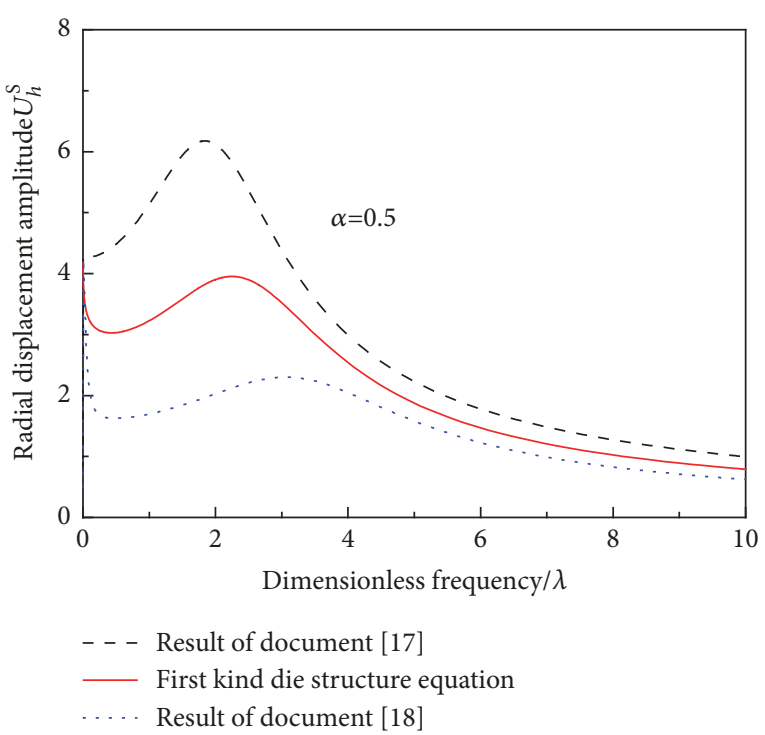

(a) Radial displacement amplitude

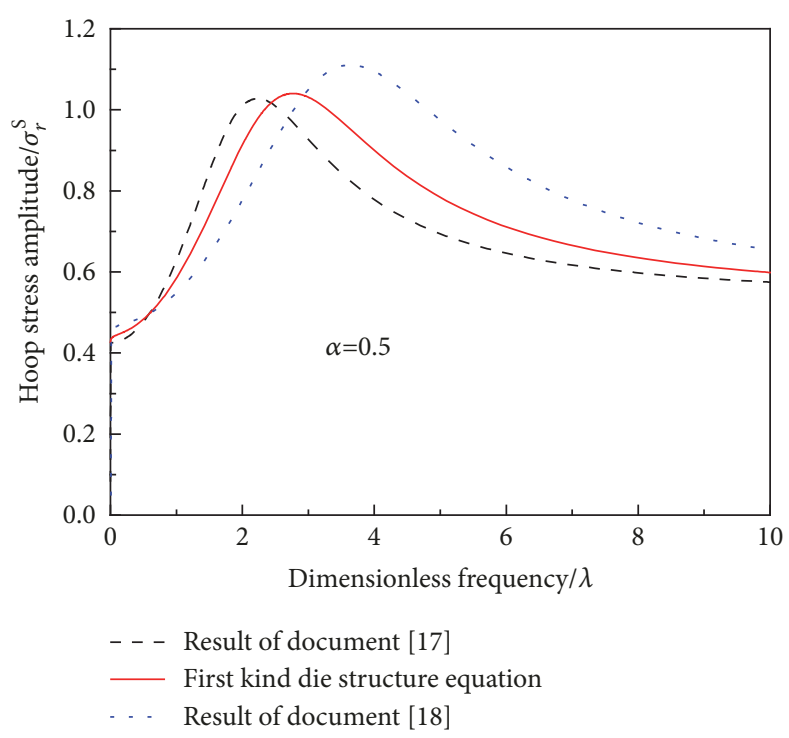

(b) Hoop stress amplitude

FIGURE 3: Comparison analysis of three models.

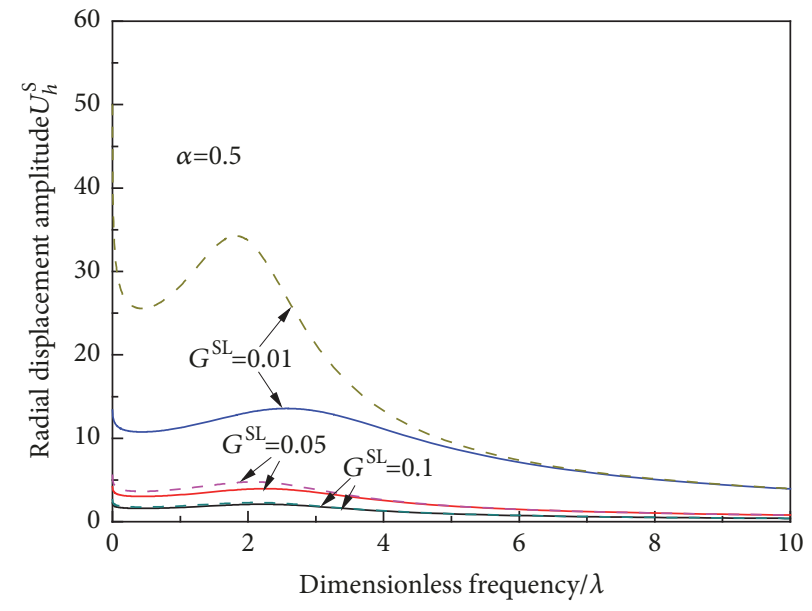

First kind die structure equation Second kind die structure equation

(a) Radial displacement amplitude

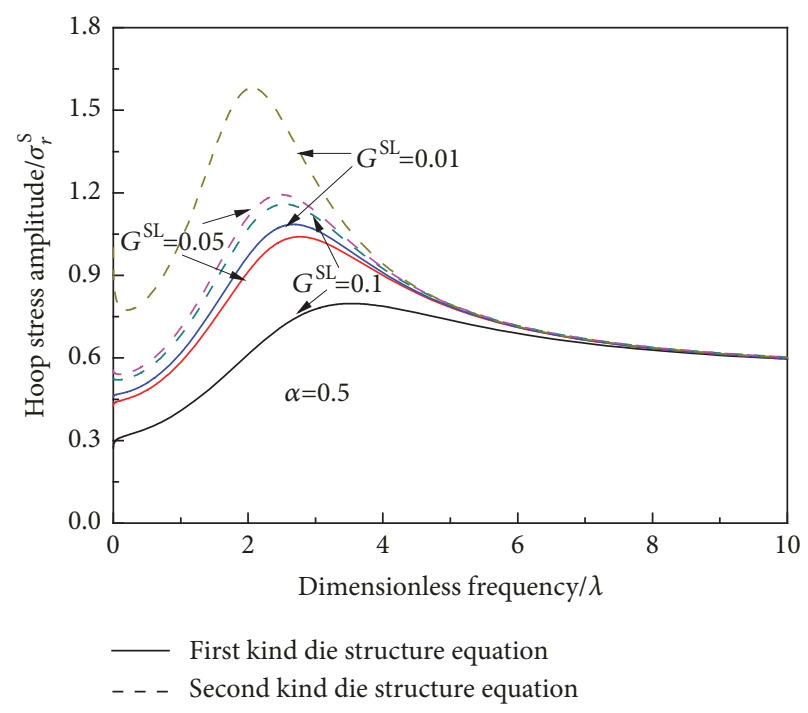

(b) Hoop stress amplitude

FIGURE 4: Influence of modulus ratio on radial displacement and hoop stress amplitude.

latter system is significant. With the increase in the modulus ratio $\lambda$, the difference in the displacement amplitude for the two models is small. The stress amplitude is clearly greater for the spherical shell die structure than for the uniform elastic medium. As the frequency increases, the difference between the two models decreases and the displacement and the stress amplitude decrease with an increase in the modulus ratio.

Figure 5 shows the effect of the changes in Poisson's ratio $v^{s}$ on the radial displacement and the amplitude of the circumferential stress. As Poisson's ratio $v^{\mathrm{s}}$ increases, the radial displacement and amplitude of the hoop stress increase gradually. The difference in the displacement and stress amplitude between the two types of die structures at high frequency is very small.

Figure 6 shows the influence of the fractional-derivative order $\alpha$ on the radial displacement and circumferential stress amplitude. It is evident that the amplitude of radial displacement decreases with an increase in the order number $\alpha$ in genera; with the increase in the frequency $\lambda$, the difference in the displacement amplitude for the two models is small. The hoop stress changed with the fractional-derivative order number $\alpha$ being interesting. Its change has a certain 


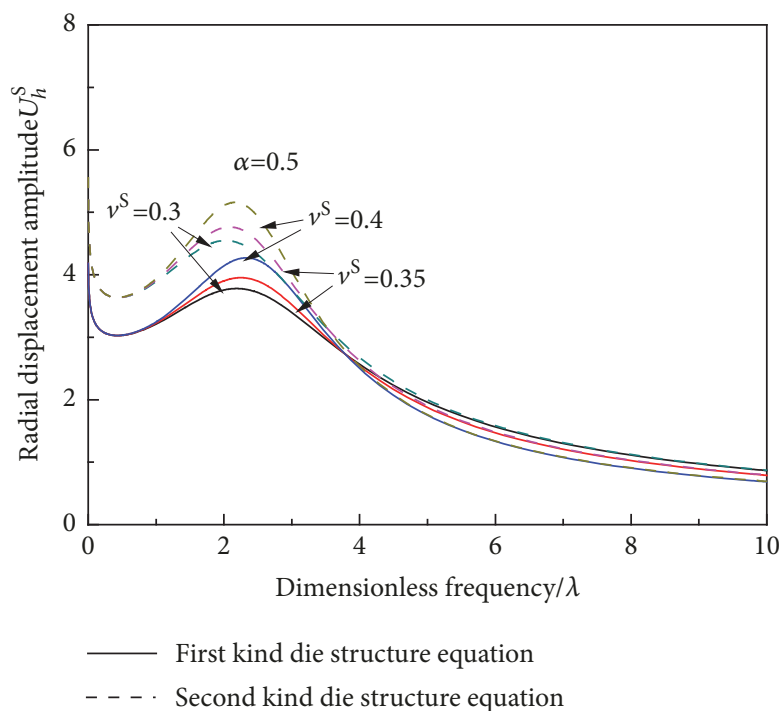

(a) Radial displacement amplitude

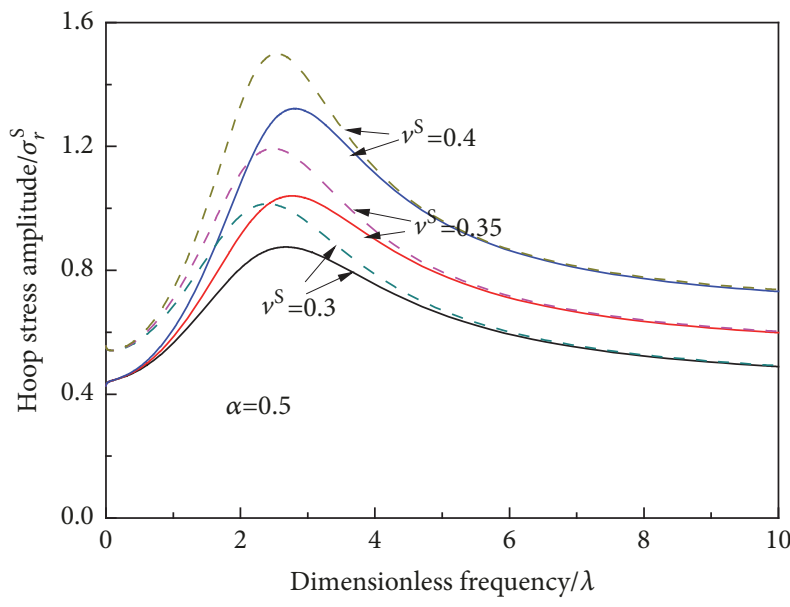

_ - First kind die structure equation

(b) Hoop stress amplitude

FIgURE 5: Influence of Poisson's ratio on radial displacement and hoop stress amplitude.

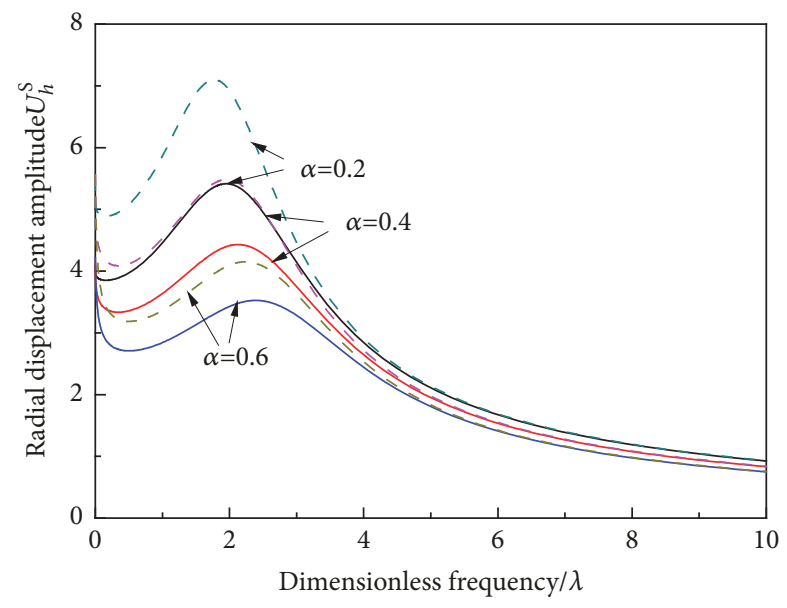

First kind die structure equation

- - - Second kind die structure equation

(a) Radial displacement amplitude

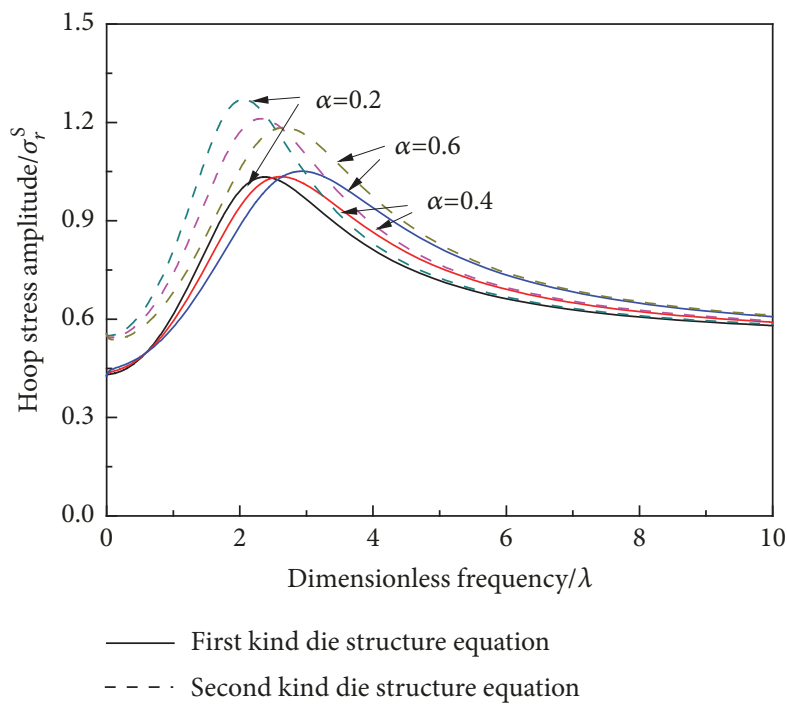

(b) Hoop stress amplitude

FIGURE 6: Influence of order on radial displacement and hoop stress amplitude.

relationship with the frequency $\lambda$ : when the frequency $\lambda$ is between 0 and 3 , the hoop stress amplitude decreases with an increase in the order number $\alpha$; when the frequency $\lambda$ is greater than 3 , the circumferential stress amplitude increases with an increase in the order number $\alpha$.

Figure 7 shows the changes in the radial displacement and circumferential stress amplitude as a result of changes in the frequency $\lambda$ when the material parameter ratio $T_{\sigma} / T_{\varepsilon}$ of the constitutive model is changed. The data indicate that the radial displacement and circumferential stress amplitude decrease as the material parameter ratio $T_{\sigma} / T_{\varepsilon}$ increases; this is caused by the increase in the impedance of the soil body.

\section{Conclusions}

In this paper, the simplified vibration characteristics of a deeply buried spherical digester in viscoelastic soil under uniform internal pressure in the frequency domain are studied using an analytics method; the effects of relative physical properties and geometrical parameters on the dynamic characteristics of the system are considered. The main conclusions are summarized as follows:

(1) Under the condition of $T_{\sigma} / T_{\varepsilon}=3$, the steady-state response of the viscoelastic soil is greater for the fractionalderivative viscoelastic soil than for the classical viscoelastic soil but less than for the classical elastic soil 


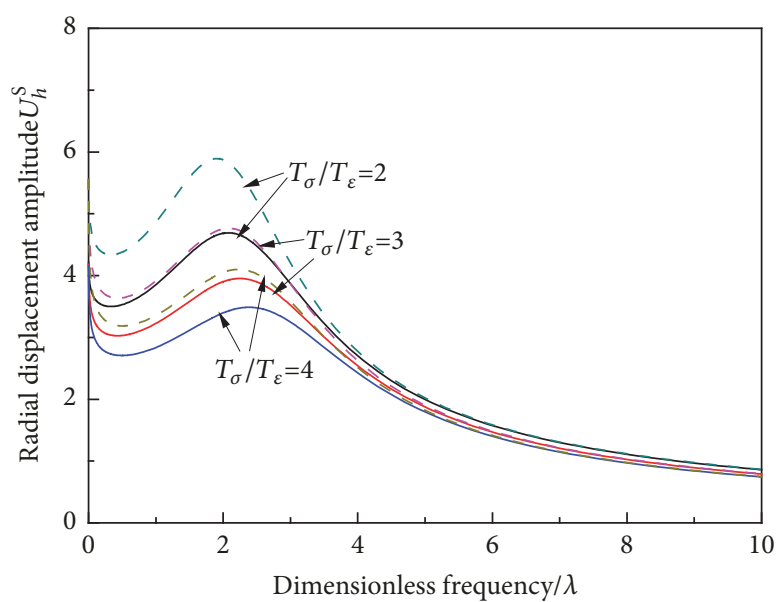

- First kind die structure equation

- - - Second kind die structure equation

(a) Radial displacement amplitude

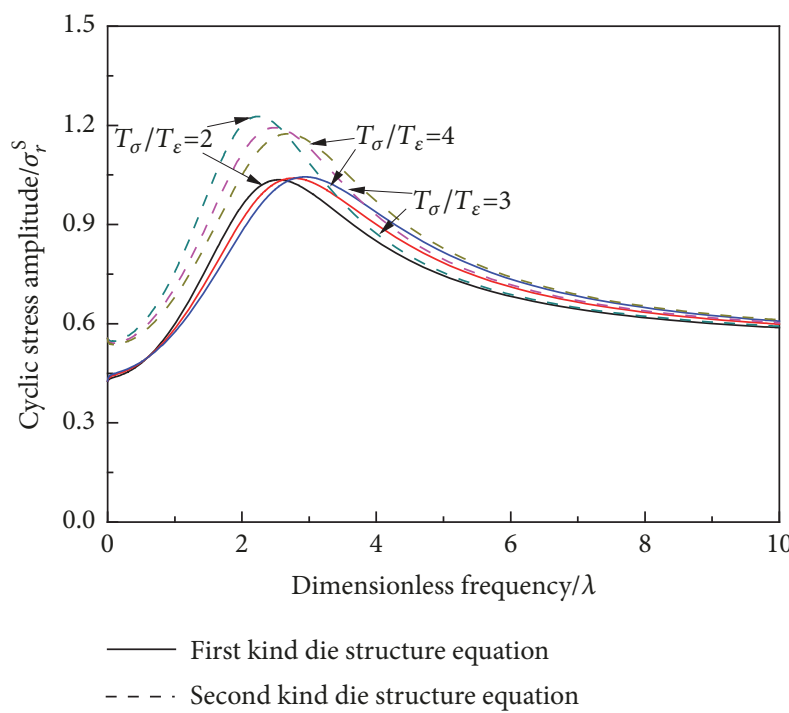

(b) Hoop stress amplitude

FIGURE 7: Influence of material parameter ratio on radial displacement and hoop stress amplitude.

(2) The steady-state response of the spherical digester is clearly greater for the spherical shell mold structure model than for the homogeneous elastic medium model

(3) When the modulus is comparatively small, the displacement amplitude is very different for the two types of mold type models and the difference between them decreases with increases in $G^{\text {SL }}$

(4) With the increase in Poisson's ratio $v^{S}$ of the soil material, the displacement amplitude and the hoop stress amplitude increases gradually

(5) With the increase in the order number $\alpha$, the radial displacement and the hoop stress amplitude decrease and the system resonance effect weakens

(6) With the increase in the material parameter ratio $T_{\sigma} / T_{\mathcal{E}}$, the radial displacement and the hoop stress amplitude decrease

\section{Data Availability}

No data were used to support this study.

\section{Conflicts of Interest}

The authors declare that they have no conflicts of interest.

\section{Acknowledgments}

The work presented in this paper was supported by the Department of Construction of Zhejiang Province Science and Technology Project (no. 2015K31), National Natural Science Foundation of China under (Grant no. 51708175), and Business Funds of Central Colleges and Universities (Grant no. 2018B00814).

\section{References}

[1] W. Gong, M. Ding, Q. Xu, and X. Jiang, "Earth pressure on pool wall and stress analysis on pool cover of underground biogas digester," Nongye Gongcheng Xuebao, vol. 27, no. 4, pp. 270-276, 2011.

[2] M. J. Forrestal and M. J. Sagartz, "Radiated pressure in an acoustic medium produced by pulsed cylindrical and spherical shells," Journal of Applied Mechanics, vol. 38, no. 4, pp. 10571060, 1971.

[3] T. A. Duffey, "Transient response of viscous-elastic and viscouselastic shells submerged in fluid media," AMSE Journal of Applied Mechanics, vol. 98, pp. 137-143, 1976.

[4] A. L. Glenn and R. E. Kidder, "Blast loading of a spherical container surrounded by an infinite elastic medium," Journal of Impact Engineering, vol. 22, pp. 984-992, 2000.

[5] Z. Qingyuan and Z. Renrui, “The dynamic response of spherical hollow chamber under explosive loading," Explosion and Shock Waves, vol. 14, no. 2, pp. 182-185, 1994.

[6] C. Gu, J. Wang, Y. Cai, L. Sun, P. Wang, and Q. Dong, "Deformation characteristics of overconsolidated clay sheared under constant and variable confining pressure," Soils and Foundations, vol. 56, no. 3, pp. 427-439, 2016.

[7] L. Guo, J. Wang, Y. Cai, H. Liu, Y. Gao, and H. Sun, "Undrained deformation behavior of saturated soft clay under long-term cyclic loading," Soil Dynamics and Earthquake Engineering, vol. 50, pp. 28-37, 2013.

[8] J. Wang, L. Guo, Y. Cai, C. Xu, and C. Gu, "Strain and pore pressure development on soft marine clay in triaxial tests with a large number of cycles," Ocean Engineering, vol. 74, pp. 125-132, 2013.

[9] C. Gu, J. Wang, Y. Cai, and L. Guo, "Influence of cyclic loading history on small strain shear modulus of saturated clays," Soil Dynamics and Earthquake Engineering, vol. 66, pp. 1-12, 2014. 
[10] C. Gu, Z. Gu, Y. Cai, J. Wang, and D. Ling, "Dynamic modulus characteristics of saturated clays under variable confining pressure," Canadian Geotechnical Journal, vol. 54, no. 5, pp. 729-735, 2017.

[11] Y. Cai, T. Wu, L. Guo, and J. Wang, "Stiffness degradation and plastic strain accumulation of clay under cyclic load with principal stress rotation and deviatoric stress variation," Journal of Geotechnical and Geoenvironmental Engineering, vol. 144, no. 5, Article ID 04018021, 2018.

[12] T. Wu, Y. Cai, L. Guo, D. Ling, and J. Wang, "Influence of shear stress level on cyclic deformation behaviour of intact Wenzhou soft clay under traffic loading," Engineering Geology, vol. 228, pp. 61-70, 2017.

[13] H. X. Gao and M. J. Wen, "Dynamic characteristics of a spherical cavity in nearly saturated viscoelastic soil," Chinese Journal of Theoretical and Applied Mechanics, vol. 44, no. 4, pp. 753-761, 2012.

[14] L.-C. Liu and X. Yang, "Analysis of vertical vibrations of a pile in saturated soil described by fractional derivative model," Rock and Soil Mechanics, vol. 32, no. 2, pp. 526-532, 2011.

[15] H. Lijun, K. Lingwei, W. Wenjun et al., "A description of creep model for soft soil with fractional derivative," Rock and Soil Mechanics, vol. 32, supplement 2, pp. 239-244, 2011.

[16] P. Weiland, "Biogas production: current state and perspectives," Applied Microbiology and Biotechnology, vol. 85, no. 4, pp. 849860, 2010.

[17] X. Changjie and W. Shiming, "Spherical wave propagation in saturated soils," Applied Mathematics and Mechanics, vol. 19, no. 3, pp. 243-252, 1998.

[18] X. Changjie and C. Yuanqiang, "Dynamic response of spherical cavity in viscous-elastic saturated soils," China Civil Engineering Journal, vol. 34, no. 4, pp. 88-92, 2001.

[19] U. Zakout and N. Akkas, "Transient response of a cylindrical cavity with and without a bonded shell in an infinite elastic medium," International Journal of Engineering Science, vol. 35, no. 13-12, pp. 1203-1220, 1997.

[20] S. P. Timoshenko and J. N. Goodier, Theory of Elasticity, McGraw-Hill, New York, NY, USA, 1977. 


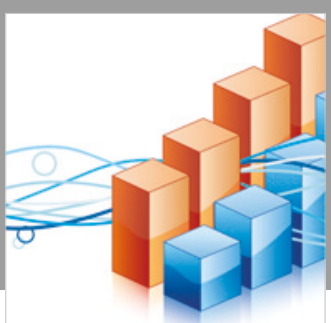

Advances in

Operations Research

\section{-n-m}
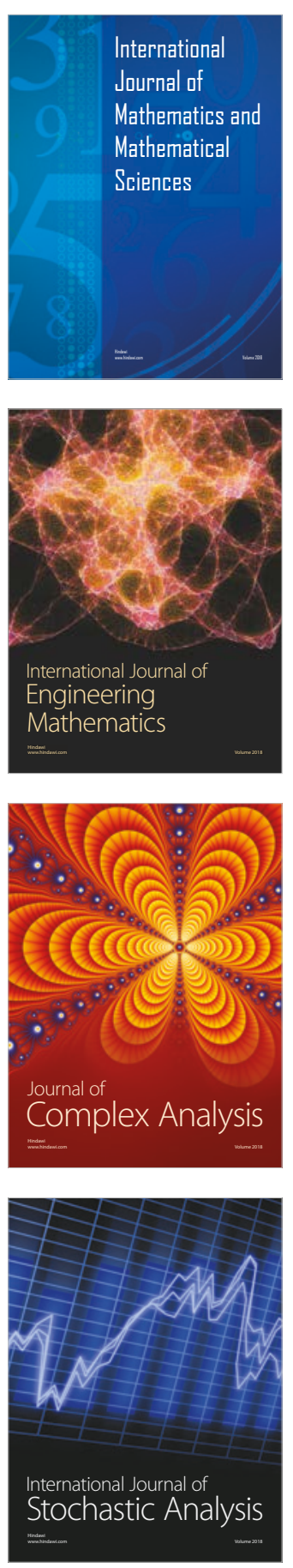
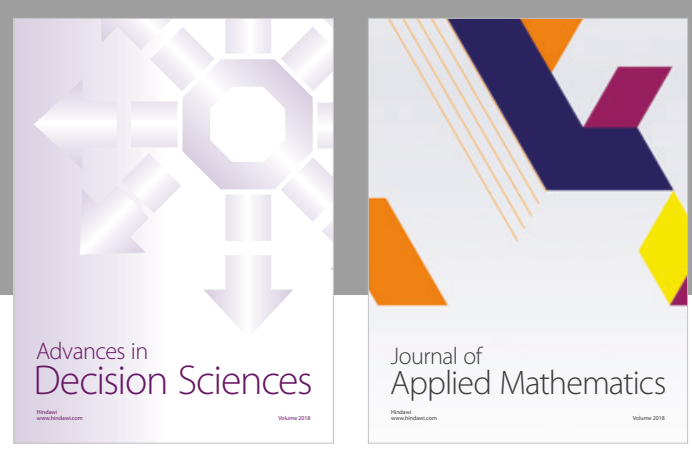

Journal of

Applied Mathematics
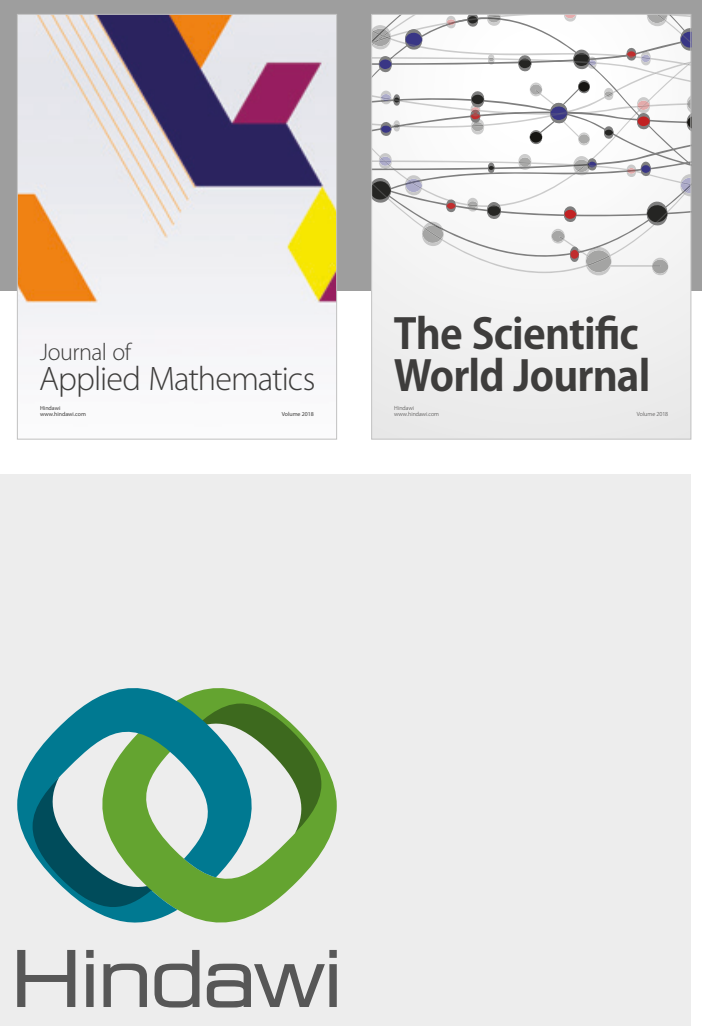

Submit your manuscripts at

www.hindawi.com

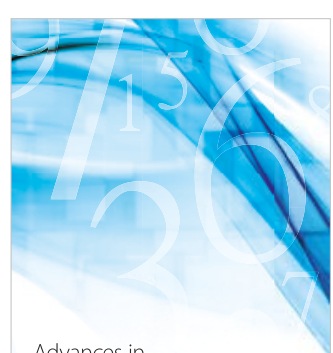

Advances in
Numerical Analysis
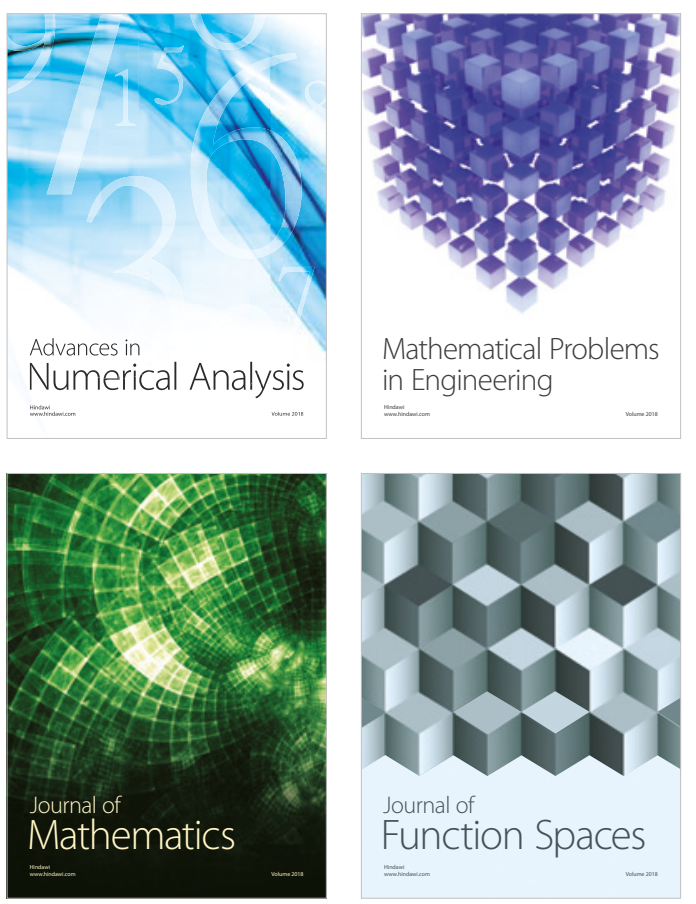

Mathematical Problems in Engineering

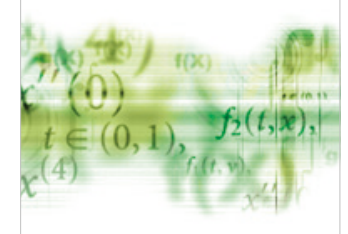

International Journal of

Differential Equations

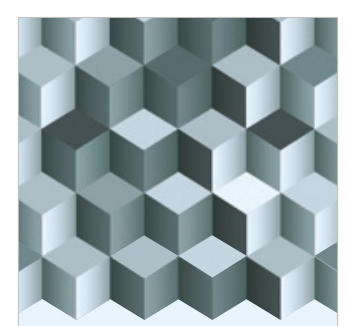

Journal of

Function Spaces
The Scientific

World Journal

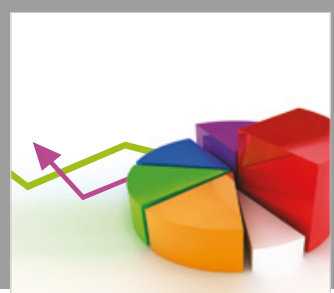

Journal of

Probability and Statistics
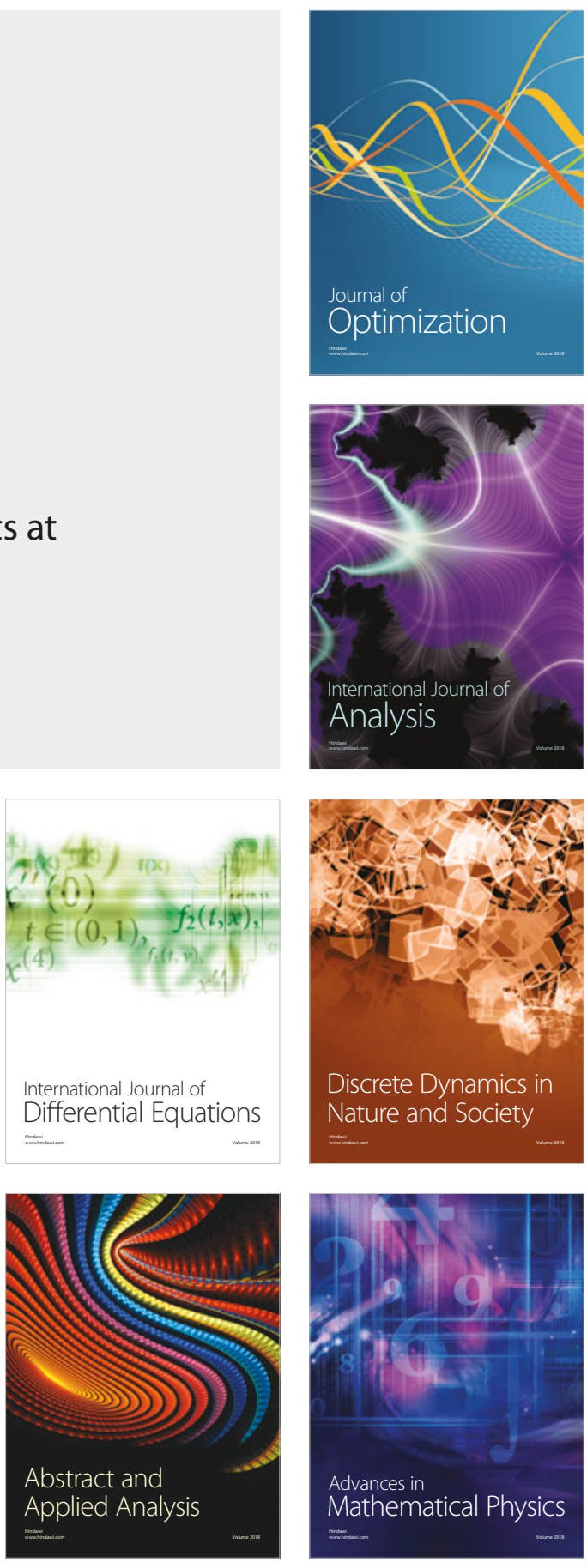